\title{
Query Expansion Based on a Personalized Mobile Search Engine-A Review
}

\author{
Anita B. Dombale ${ }^{1}$, Suhas M. Patil ${ }^{2}$ \\ ${ }^{1,2}$ Computer Engineering Department, K J College of Engineering \& Management Research, Pune, India
}

\begin{abstract}
Day by day internet is becoming very crowdies with no. of web pages and no. of its users. So due to that it is becoming difficult to find exact resultant web pages for particular query in web search. Though recent developments on web search technologies, still for many situations users obtains the non-relevant search results from the search engines. A solution on this problem is personalization. Second thing, limitation of mobile search engine are, small parts of mobile like small screen, tiny keypad. due to these limiting factors user has to give short and ambiguous query. and in this condition also for getting relevant result for query, personalization is needed. Personalization means creating user profile inbuilt form user's previous clickthrough collector. This paper focuses on the survey of many efficient personalized Web search approaches and personalized mobile search approaches which were proposed by many authors.
\end{abstract}

Keywords: Clickthrough collector, reranking, personalization, user profile, concepts

\section{Introduction}

Now a days commonly used task on the internet is web searching. The limitation of web search engine is 1)Lack of user adaption,2)Retrieve results based on web popularity rather than user's interests 3)users typically view only the first few pages of search results. So due to these limitation relevant results beyond first few pages have a much lower chance of being visited. So personalization is needed.

It's a mobile search engine. For mobile search small form of factors are the limiting factors i.e. small keypad, small screen. So due to these limiting factors user has to give short query for mobile search but at the same time relevant results are expected.

We can say user has got irrelevant documents or user has got failure when search engines returns a results which are not useful to the users or do not meet searched query expectation. e.g. Suppose a housewife and a Mobile user take "apple" in different meaning for search context. Suppose A housewife want to know the apple price in market. While a mobile user is interested in services related to apple mobile. Many researchers worked to determine the query ambiguity. A typical search engine provides same results for both users though their expected results are different. Many researchers worked to determine the query ambiguity. Therefore, the need arises to have personalized web search system.

Personalization aims to tailor search results[12] to individuals depend on knowledge of user's interests, find relevant documents and arrange them on top of the result list, filter irrelevant search results. The approaches [12]for applying user profile are as shown in fig. 1
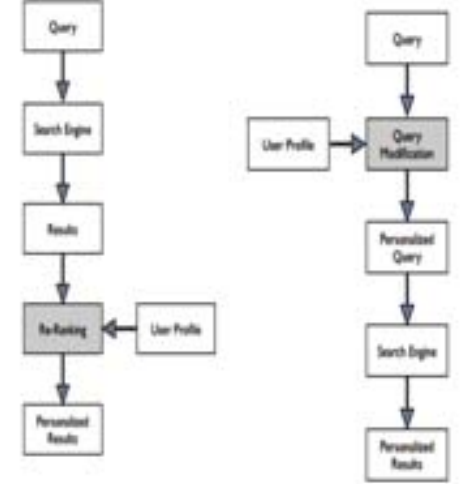

Part of retrieval process:

Personalcation buit info the search engine

\section{Result Re-ranking:}

User Profle used to re-rank search results retumed from a standard, non-personalized search engines
Query Modification:

User profile affects the $d$ the information need submitied representation

Figure 1: Approaches for applying user Profile

This Paper describes the various personalized web search approaches and mobile search approaches proposed by many authors.

\section{Literature Review}

L. Fitzpatrick and M. Dent [1] developed a personalized web search, in which user has to give all their information during training period information user has to give is like user's age, interest etc. second method in which user has to give feedback .giving feedback means telling which links are relevant to user and which are irrelevant. user tells this by rating from 1 (very bad) to5(very good). This approach had a lot of limitations. Creating profile explicitly means, it may happen that sometimes user may give wrong information, which hampers user profile and also it is a time taking process

Amir Zidia,*, Amna Bouhanab, Mourad Abeda, Afef Fekihc7[2]proposed model using the Case Base Reasoning (CBR) tool called as ontology-Based Personalized Retrieval model. 


\section{International Journal of Science and Research (IJSR) \\ ISSN (Online): 2319-7064}

Index Copernicus Value (2013): 6.14 | Impact Factor (2015): 6.391

The proposed system combines the advantages of two methods, a content-based method (ontology) for representing data and a case-based method (CBR) making search process personalized and for giving users with alternate documents recommendations.

Jonghyun Hana,1, Hedda R. Schmidtke b,*, Xing Xie c, Woontack Woo d[3] proposed a search engine that depends on context that shows user context in a knowledge-based context model, implemented in a hierarchy structure with granularity information. Based on semantic relevance, search results are ordered.

K.W.T. Leung, D.L. Lee and Wang-Chien Lee [4] extracts user's preferences in the form of concepts from user's previous clickthrough data.

Concepts are content concepts and location concepts. Along with location concepts from click through data GPS are used as second support for location concepts in PMSE. The client privacy is also considered.

Zhicheng Dou, Ruihua Song, Ji-Rong Wen, and Xiaojie Yuan[6] studied questions like whethere personalization concepts is equally effective for different types of queries, for different users and for different search contexts.\& provide some findings. Authors presented a large-scale evaluation framework of query log for personalized search and then evaluate five algorithm which are personalized search algorithm. out of 5 algorithm two are click based and remaining are topical interest based. authors used 12-day query logs of Windows Live Search. By analyzing the results, authors told that personalization doesn't work equally for all types of conditions. It shows a significant improvement over generic Web search for some queries, while it has harm on query performance for some situations. authors proposes click entropy measurement for marking whether a query should be personalized.

K R Remesh Babua,*, Philip Samuelb[7] proposes method in which, to identify users search preferences, a concept network is created.from user's previous search clist of concepts is taken called as concept network. This technique helps to fetch the web pages related to the context in which the user needs information. When user searched something new Genetic Algorithm (GA) is used. for comparing the user's concept network with other user's concept network for similarity,GA is used which helps to get a better search in their area of interest.

P. Palleti H. Karnick and P. Mitra [8] introduced probabilistic query expansion for personalized web search. The Web is full of data and search engine suggests professional ways to web for getting positive results. Common search engines provides results to user without considering their purpose of the query. Author's Personalized Web search system gives positive results to user by considering user's interest. authors developed a system applied at proxy which considers user profile with the use of collaborative filtering and changes user's interest . probabilistic correlations among query terms and document terms are find out and which is used as a user profile for giving personalized search results. Experimental outcomes shows that author's system is very effective and efficient.

Chen Ding, J.C. Patra and Fu Cheng Peng [9]introduced personalized web search with self organizing map.generic web search engine gives same results to many users though their needed results are different. Personalized Web search performs search as per user preference. for reducing of time on browsing irrelevant documents, this paper suggests a new concept called intelligent Personal Agent for Web Search (PAWS) which cleverly utilizes the self organizing map (SOM) as the user's profile and therefore, can give high quality answer set to the user.

Based on the conceptual modeling of the user's navigational behavior S. Acharyya, J. Ghosh[10] proposed a general personalization model. The model involves whatever page user visits are mapped to a topic or concept by which tree hierarchy is created. and parameters of a semi- Markov process defined on this tree based on the observed user paths are estimated. Finding semantic characteristics the context is performed manually in Markov models based work. semantic similarity measure is not exploited for improving the prediction process, except for generalizations/specializations of the ontology terms.

O. Shafiq, R. Alhajj and J.G. Rokne [11] introduced community aware personalized Web search. Searching for correct information over the Web is difficult. In the world of high speed internet, elevated capacity networks, and interactive Web applications, it has become more easier to publish data online. A huge amount of data is published over the internet; every data is in the form web pages, news, blogs and other material, etc. In the same way, for search engines like Google and Yahoo, it becomes slightly hard to discover the accurate information, i.e., as per user's preferences; search results for same query by many users would be different in priority for different users. In this paper, the authors proposed a technique to display results to user according to user's interest and context of a user. according to the individual interests and context of users. With the purpose of finding out personal interest and context, this paper follows a distinctive method of (a) finding out behaviors of a user of his/her social-network, (b) finding out what information does the social networks (i.e., friends and community) present to the user. Based on this information, the authors have come up with a methodology which considers the information about social networks and search outputs are prioritized.

\section{Problems and Directions}

Many Personalized Web search approaches and mobile search approaches have been discussed in the literature survey. The following directions will help the researchers and guide them to develop the best Personalized Web search and Personalized Mobile search approach.

\section{A) Construction of User Profile}

For some proposed systems user profiles are created manually /defined explicitly by user. So user profiles can be created automatically implicitly.

\section{B) Security of User Profiles}




\section{International Journal of Science and Research (IJSR) \\ ISSN (Online): 2319-7064}

Index Copernicus Value (2013): 6.14 | Impact Factor (2015): 6.391

For many proposed systems user profile security is a major problem.

\section{c) Location concept Extraction}

Location concepts can be extracted from clickthrough data and by GPS System.

\section{Conclusion}

Day by day Internet is becoming very crowdies with no. of web pages and no. of its users. So due to that it is becoming difficult to find exact resultant web pages for particular query in web search. Though recent developments on web search technologies, still for many situations users obtains the non-relevant search results from the search engines As a solution on this problem is personalization. Second thing, limitation of mobile search engine are, small parts of mobile like small screen, tiny keypad. due to these limiting factors user has to give short and ambiguous query. and in this condition also for getting relevant result for query, personalization is needed. Personalization. This paper Focuses on the various techniques proposed by different authors for getting relevant results to each user for their respective query.

\section{Acknowledgement}

The authors would like to thank the researchers as well as publishers for their resources available and teachers for their guidance

\section{References}

[1] L. Fitzpatrick and M.Dent, "Automatic Feedback Using Past Queries: Social Searching”, Proc.20th Conf., 1997.

[2] Amir Zidia,*, Amna Bouhanab, Mourad Abeda, Afef Fekihc7,"An ontology-based personalized retrieval model using case base Reasoning", 18th International Conference on Knowledge-Based and Intelligent, Information \& Engineering Systems - KES2014.

[3] Jonghyun Hana, 1, Hedda R. Schmidtke b,*, Xing Xie c, Woontack Woo d,"Adaptive content recommendation for mobile users: Ordering recommendations using a hierarchical context model with granularity", Pervasive and Mobile Computing 13 (2014) 85-98

[4] Kenneth Wai-Ting Leung, Dik Lun Lee, and WangChien Lee," Personalized W eb Search with Location Preferences", IEEE Transactions On Knowledge And Data Engineering, Vol. 25, No. 4, April 2013.

[5] K.W.T. Leung, D.L. Lee and Wang-Chien Lee, "Personalized Web search with location preferences", IEEE 26th International Conference on Data Engineering, pp. 70 I - 712, 2010

[6] Zhicheng Dou, Ruihua Song, Ji-Rong Wen, and Xiaojie Yuan," Evaluating the Effectiveness of Personalized Web Search", IEEE TRANSACTIONS ON KNOWLEDGE AND DATA ENGINEERING, VOL. 21, NO. 8, AUGUST 2009

[7] K R Remesh Babua, *, Philip Samuelb," Concept Networks for Personalized Web Search Using Genetic Algorithm" ,International Conference on Information and Communication Technologies (ICICT 2014)
[8] P. Palleti, H. Karnick and P. Mitra, "Personalized Web Search Using Probabilistic Query Expansion," International Conferences on Web Intelligence and Intelligent Agent Technology Workshops (IEEE/WIC/ACM), Pp. 83 - 86, 2007.

[9] Chen Ding, J.C. Patra and $\mathrm{Fu}$ Cheng Peng“" Personalized Web search with self-organizing map," The 2005 IEEE International Conference on etechnology, e-Commerce and e-Service (EEE '05),Pp. $144-147,2005$

[10] S. Acharyya, J. Ghosh, "Context-Sensitive Modeling of Web Surfing Behaviour Using Concept Trees", in Proc. of the 5th WEBKDD Workshop, Washington, August 2003.

[11] O. Shafiq, R. Alhajj and J.G. Rokne, "Community Aware Personalized Web Search," International Conference on Advances in Social Networks Analysis and Mining (ASONAM), Pp. 351 - 355,2010.

[12]Kapil Goenka , "Mobile Web Search Personalization Using Ontological User Profile" http://cobweb.cs.uga.edu/ budak/thesis/kapil thesis.pdf

[13] K.W.-T. Leung, D.L. Lee, and W.-C. Lee, "Personalized Web Search with Location Preferences," Proc. IEEE Int'l Conf. Data Mining (ICDE), 2010. 